\title{
Plasmonic Hot Electron Induced Layer Dependent Anomalous Fröhlich Interaction in InSe
}

\section{Mahfujur Rahaman ( $\nabla$ engr.maheee@gmail.com )}

Chemnitz University of Technoloy https://orcid.org/0000-0002-3912-8509

Muhammad Aslam

Chemnitz University of Technology

Lu He

Chemnitz University of Technology

Teresa Madeira

Chemnitz University of Technology

Dietrich Zahn

Chemnitz University of Technology, Institute of Physics https://orcid.org/0000-0002-8455-4582

\section{Article}

Keywords:

Posted Date: February 22nd, 2021

DOI: https://doi.org/10.21203/rs.3.rs-264548/v1

License: (c) (1) This work is licensed under a Creative Commons Attribution 4.0 International License. Read Full License

Version of Record: A version of this preprint was published at Communications Physics on July 30th, 2021. See the published version at https://doi.org/10.1038/s42005-021-00674-5. 


\title{
Plasmonic Hot Electron Induced Layer Dependent Anomalous Fröhlich Interaction in InSe
}

\author{
Mahfujur Rahaman*, Muhammad Awais Aslam, Lu He, Teresa I. Madeira, \\ and Dietrich R. T. Zahn \\ Semiconductor Physics, Chemnitz University of Technology, 09107 Chemnitz, Germany \\ mahfujur.rahaman@physik.tu-chemnitz.de
}

\begin{abstract}
InSe is one of the most promising two-dimensional (2D) materials for electronic and optoelectronic applications because of its favourable bandgap and superior electron mobility compared to other layered semiconductors. However, due to the polar nature of InSe, Fröhlich interaction plays an important role in electrical transport, which becomes more significant in reduced dimensionality. Until now, it is not yet known how the dimensionality influences the strength and nature of the Fröhlich polaronic effect in InSe. Here, we report on layer dependent anomalous Fröhlich interaction in InSe from bulk to monolayer with the aid of plasmonic hot electron doping. When excited near the localized surface plasmon resonance, plasmonic nanostructures produce highly energetic electrons (known as hot electrons), which can be captured by a semiconductor such as InSe at the interface. These electrons then couple to the polar optical phonons via the Fröhlich interaction in InSe. With the aid of the strong plasmonic field, the Fröhlich interaction enabling us to monitor the polar phonons in conventional Raman measurements. We prepared nanostructures with three different metals ( $\mathrm{Ag}, \mathrm{Au}$, and $\mathrm{Al}$ ) using nanosphere lithography on InSe to study the hot electron doping effect by means of Raman spectroscopy. A finite element method simulation was used to understand the coupling between the plasmonic nanostructures and InSe. We observed that the intensity of polar LO phonon modes initially increases gradually with decreasing layer number and then drops drastically from $7 \mathrm{~L}$ to $6 \mathrm{~L}$, i.e. at the thickness where the transition from quasi-direct to indirect bandgap occurs at room temperature. Additionally, a gradual decrease of intensity of the polar modes with decreasing layer thickness below this transition point is observed, which is due to the increasing indirect bandgap nature of InSe suggesting reduced Fröhlich coupling. Our results shed light on fundamental understanding of Fröhlich interaction in InSe, which is crucial for electronic and optoelectronic applications of this promising 2D material.
\end{abstract}

Keywords: 2D materials, InSe, Fröhlich interaction, plasmonic hot electron, layer dependent Raman spectroscopy 


\section{Introduction}

Among all 2D semiconductors, InSe (a member of metal monochalcogenides, MMC) has emerged as an outstanding candidate for post-silicon electronic devices. It possesses a high electron mobility $\left(\sim 1 \mathrm{O}^{3} \mathrm{~cm}^{2} \mathrm{~V}^{-1} \mathrm{~s}^{-1}\right)$ due to its small effective mass $\left(\sim 0.14 \mathrm{~m}_{\mathrm{e}}\right)$ at room temperature (RT) ${ }^{1}$. Even though 2D black phosphorus (BP) has a comparable mobility ${ }^{2}$, it is extremely unstable in environmental conditions 3 , which limits the possibility of practical applications. The large surface-to-volume ratio and the atomic thickness of $2 \mathrm{D}$ materials make them ideal for electrostatic control and significant device downscaling for high-density integration 4 . Moreover, the ultraclean, dangling bond free smooth surfaces of these materials are less sensitive to carrier scattering. They can thus outperform existing silicon devices in the regime of scaling limitation. Therefore, InSe provides a promising playground for studying low dimensional phenomena and for developing high mobility nanoelectronics 4,5 .

One of the limiting factors of intrinsic mobility are the phonons, quanta of lattice vibration perturbing the electron potential in a lattice. In polar materials, optical phonons couple strongly with elementary charges due to long range Coulomb interactions induced by the macroscopic polarization field resulting from the atomic displacement, known as Fröhlich interaction ${ }^{6}$. Therefore, scattering with optical phonons limits the mobility in 2D semiconductors at RT significantly 7,8. Intriguingly, 2D Fröhlich interaction is markedly different from its bulk analogue as reported in a recent theoretical work 9. The Fröhlich interaction in 2D materials diverges from the $3 \mathrm{D}$ one at zero phonon-momentum limit creating van Hove singularities, where it transforms into a very large finite value compared to the standard electron-phonon coupling (EPC). As a consequence, due to the reduced dielectric screening, the enhanced macroscopic field created by the phonon assisted polarization density may lead to a stronger EPC in ultra-thin materials resulting in a layer-dependent Fröhlich potential and hence device performance. Therefore, the question of how the dimensionality influences the Fröhlich interaction in InSe has great significance and importance for both fundamental understanding and device application. Very recently, Ma et al. 7 and Li et al. ${ }^{10}$ laid the theoretical ground for understanding the 2D model of Fröhlich potential and layer-dependent Fröhlich interaction in InSe by means of intrinsic carrier mobility. To the best of our knowledge, no experimental investigation of layer-dependent Fröhlich interaction in 2D semiconductors, in particular for InSe, has been reported until now.

To accomplish this goal, a systematic investigation of layer-dependent Fröhlich interaction in InSe via plasmonic hot electron doping was performed. Plasmonic nanostructures have outstanding light-trapping and electromagnetic field-confining properties and can generate hot electrons with energies up to $4 \mathrm{eV}$ when excited at localized surface plasmon resonance (LSPR) ${ }^{11}$. 
These highly energetic electrons can escape from the plasmonic structures and be collected by the conduction band (CB) of adjacent semiconductors depending on the energy barrier at the interface. The small Schottky barrier $(0.1-0.9 \mathrm{eV})$ of InSe with noble metals is, therefore, an ideal system for the collection of hot electrons from the plasmonic structures ${ }^{12,13}$. In polar semiconductors, the electric field carried by the free carrier plasmons (here the hot electrons collected by the $\mathrm{CB}$ of InSe) interact with the long range macroscopic field of the longitudinal optical phonons (LO) breaking the Raman selection rules ${ }^{14}$. Therefore, Raman spectroscopy can directly measure coupled modes ${ }^{15}$ enabling the investigation of the polar LO phonons via plasmonic hot electrons doping as a function of InSe layer number.

InSe studied in this work belongs to the commercially available rhombohedral $\gamma$-type crystal structure with a $\mathrm{ABC}$ stacking sequence and no centrosymmetry ${ }^{16}$. The unit cell consists of three tetra-atomic layers in which, each tetra-atomic layer (Se-In-In-Se) creates the monolayer of InSe ${ }^{17}$. The thickness of each monolayer is $0.8 \mathrm{~nm}$. Bulk InSe is a direct bandgap ( $\mathrm{E}_{\mathrm{g}}$ ) semiconductor ${ }^{18}$. However, when thinned down to below $20 \mathrm{~L}$ the valence band (VB) maxima forms a 'Mexican hat' like structure centered at the $\Gamma$ point with the energy difference between the two maxima and the center minimum smaller than the thermal activation energy at room temperature (RT) ${ }^{19}$. Therefore, it remains a quasi-direct bandgap semiconductor down to a certain thickness at RT. Like other layered semiconductors, its bandgap can be tuned upward by as large as $\sim 1.0 \mathrm{eV}$ from $1.2 \mathrm{eV}$ in bulk to $2.1 \mathrm{eV}$ in monolayer ${ }^{18}$. One notable characteristic of InSe is that it has large optical anisotropy near $\mathrm{E}_{\mathrm{g}}$ : the electronic transitions associated with polarization of incident light parallel to the $c$ axis $(\boldsymbol{E} \| \boldsymbol{c})$ are two order of magnitude larger than that perpendicular to $c$ axis $(E \perp c){ }^{18}$. In contrary the electronic transition at around $2.42 \mathrm{eV}\left(\mathrm{E}_{1}\right.$ electronic transition) is optically allowed in the normal incident geometry $(\boldsymbol{E} \perp \boldsymbol{c})$. The $\mathrm{E}_{1}$ gap also exhibits a layer dependence varying from $2.42 \mathrm{eV}$ to $3.04 \mathrm{eV}$ from bulk to monolayer, respectively ${ }^{18}$.

Being a member of the $R 3 \mathrm{~m}$ space group with four atoms per monolayer, there are twelve normal modes of vibrations in $\gamma$-InSe. Group theory predicts $4 \mathrm{~A}_{1}$ and $4 \mathrm{E}$ modes at the $\Gamma$ point for $\gamma$-InSe and apart from the acoustic $\mathrm{A}_{1}$ and $\mathrm{E}$ ones all other modes are Raman and infrared active, but with quite very different intensities ${ }^{20}$. Among them, two modes with $\mathrm{A}_{1}$ and $\mathrm{E}$ symmetry are strongly polar and therefore lead to strong infrared absorption. However, at resonant Raman conditions, when the excitation energy matches the $\mathrm{E}_{1}$ electronic transition, the $q$ dependent $\mathrm{A}_{1}$ (LO) mode around $200 \mathrm{~cm}^{-1}$ is enhanced dramatically ${ }^{21}$. When performing Raman measurements far below the $\mathrm{E}_{1}$ transition in a plasmonic environment, in which the excitation energy approaches the LSPR (surface enhanced Raman scattering (SERS) conditions), we were able to observe three 
phenomena. These are: (i) appearance of two polar modes $A_{1}$ (LO) and $E$ (LO) around $200 \mathrm{~cm}^{-1}$ and $210 \mathrm{~cm}^{-1}$ for InSe, (ii) the intensity of both polar modes are layer dependent with the $\mathrm{E}$ (LO) mode having a stronger sensitivity to layer thickness, and (iii) a drastic intensity reduction of the polar modes for the InSe layer number decreasing from $7 \mathrm{~L}$ to $6 \mathrm{~L}$, which correlates directly to the transition point from quasi-direct to indirect bandgap at RT 19,22. Using the combination of different experiments on a large number of samples ( $>20$ ), we confirm that the appearance of both polar LO modes is due to the Fröhlich interaction via plasmonic hot electron doping. The experimental evidence was further confirmed by finite element method simulations of the plasmonic coupling between metal nanostructures and InSe using COMSOL.

Our work provides the first clear picture of layer-dependent Fröhlich interaction induced EPC in InSe and offers great prospect in the design and optimization of next generation high mobility nano-electronics/optoelectronics. It also opens a new prospective for very interesting applications like photovoltaics ${ }^{23}$ and photocatalysis 24 .

\section{Experimental details}

\section{Sample preparation}

The InSe material used in this work is undoped and was purchased from 2D Semiconductors, USA. Thickness dependent InSe samples were prepared using a PDMS assisted deterministic dry transfer method onto $\mathrm{SiO}_{2}(300 \mathrm{~nm}) /$ silicon substrates ${ }^{25}$. After transferring the flakes, selfassembled monolayers of polystyrene spheres (PS) of $400 \mathrm{~nm}$ size were used for nanosphere lithography (NSL) creating a hexagonally packed metal (Ag, Au, Al) arrays of nanotriangles (NTs) with $\sim 120 \mathrm{~nm}$ side length and $\sim 50 \mathrm{~nm}$ height on the sample surface. Details of the deposition technique can be found elsewhere ${ }^{26}$.Prior to the dry transfer, photoluminescence (PL) spectra were acquired for each flake for thickness determination.

\section{PL and Raman measurements}

A Horiba Xplora plus system equipped with an electron multiplying charge couple detector (EMCCD) was used for both PL and Raman measurements at normal incidence. PL measurements were carried out using a $532 \mathrm{~nm}$ ( $2.33 \mathrm{eV})$ excitation. Raman spectra of InSe were acquired in backscattering geometry using $532 \mathrm{~nm}(2.33 \mathrm{eV}), 638 \mathrm{~nm}(1.94 \mathrm{eV})$, and $785 \mathrm{~nm}(1.58$ $\mathrm{eV}$ ) excitations and a $1200 \mathrm{l} / \mathrm{mm}$ grating was used to disperse the Raman signals onto the EMCCD. A 100 x, 0.9 NA objective was used to excite and collect the signals for both PL and Raman measurements. The laser powers of $100 \mu \mathrm{W}$ and $\sim 10 \mu \mathrm{W}$ were employed at the sample surface for 

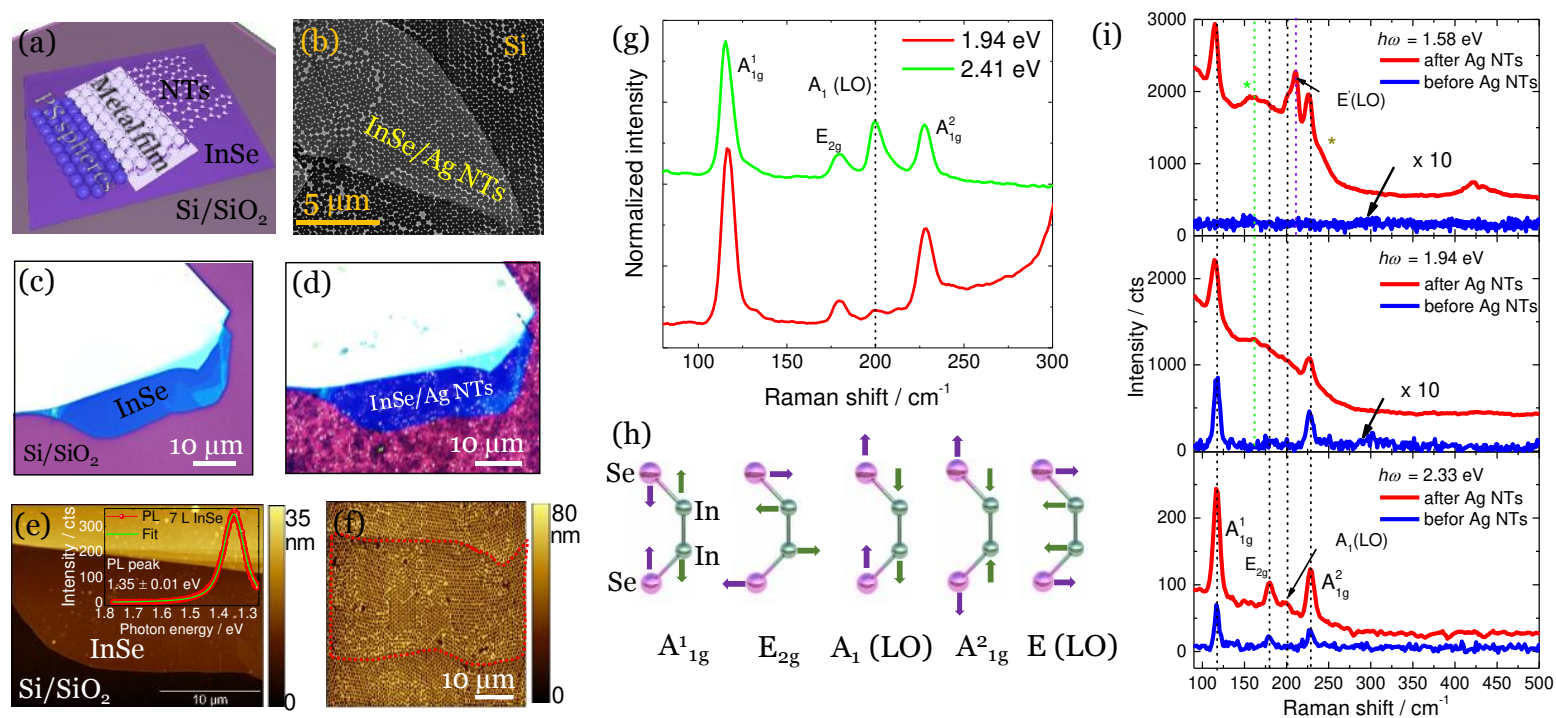

Figure 1. (a) Schematic representation of the sample preparation. Polystyrene spheres of $400 \mathrm{~nm}$ size were used for NSL. Ag, Au, and Al were used for NT formation. (b) A typical SEM image of the InSe sample after Ag NT deposition. Silicon with native oxide was used as substrate for SEM to avoid charging effects. (c), (d) optical images and (e), (f) AFM topographies of one typical InSe sample with different thicknesses before and after NSL with Ag, respectively. Inset of (e) displays the PL spectra of 7L InSe with a Voigt fitting. The red dashed lines in (f) marks the area of the thin flake as shown in the optical images. (g) Resonant and non-resonant Raman spectra acquired with $2.41 \mathrm{eV}$ and $1.94 \mathrm{eV}$, respectively, of 7L InSe. Acquisition time for the Raman measurements were $10 \mathrm{~s} \times 2$ and $30 \mathrm{~s} \times 10$, respectively, and the laser power in both measurements were $\sim 100 \mu \mathrm{W}$. (h) Elongation patterns of the phonon vibrations visible in the Raman spectra. (i) Raman spectra of 7L InSe before and after NSL with Ag acquired with $1.58 \mathrm{eV}, 1.94 \mathrm{eV}$, and 2.33 $\mathrm{eV}$ excitations. The broad spectral feature between 150 and $190 \mathrm{~cm}^{-1}$ is due to the presence of $\mathrm{E}_{2 \mathrm{~g}}$, and contribution of $\mathrm{In}_{2} \mathrm{O}_{3}$ (green dashed line and asterisk). The grey asterisk indicates a feature assigned to amorphous Se. Acquisition time for the measurements were $5 \mathrm{~s} \times 2$ and the laser power was $10 \mu \mathrm{W}$.

PL and Raman measurements, respectively. The spectral acquisition times were different for different measurement sets and stated in the respective result sections below.

Resonant Raman spectra of multilayer InSe at $\mathrm{E}_{1}$ transition (2.42 eV) were acquired under $514.7 \mathrm{~nm}(2.41 \mathrm{eV})$ excitation using a Horiba LabRam HR8oo, a symphony CCD detector, and $600 \mathrm{l} / \mathrm{mm}$ grating. A $100 \mathrm{x}, 0.9 \mathrm{NA}$ objective was used to excite and collect the signal and the laser power was $100 \mu \mathrm{W}$ at the sample surface.

Temperature dependent Raman measurement were carried out using a liquid nitrogen cooled Linkam stage and $1.58 \mathrm{eV}$ laser excitation in the Xplora plus system with the aid of a $50 \mathrm{x}$, 0.45 NA objective. 


\section{Finite Element Method Simulation}

Finite element method (FEM) simulations of InSe/metal NTs were performed using COMSOL multiphysics 5.6 in $3 \mathrm{D}$ platform in the wavelength domain. The dimension of the NTs were set according to the experimental conditions (side length: $120 \mathrm{~nm}$, height: $50 \mathrm{~nm}$ ). In order to avoid a singularity problem and to have an optimal approach to real conditions, edges and corner of the NTs were rounded with a radius of curvature of $5 \mathrm{~nm}$. The $7 \mathrm{~L} \mathrm{InSe}$ on $300 \mathrm{~nm} \mathrm{SiO} 2$ is modelled as a $7 \mathrm{~nm}$ thick InSe layer. The optical constant of $\mathrm{InSe}{ }^{27}, \mathrm{Ag}^{28}, \mathrm{Au}^{28}$, and $\mathrm{Al}{ }^{29}$ were taken from the literature.

\section{SEM and AFM measurements}

SEM images were taken using a scanning electron microscope (FEI Nova NanoSEM 200) with an accelerating voltage of $10 \mathrm{kV}$, and $5 \mathrm{~mm}$ working distance in immersion mode (highresolution mode). AFM topography images were acquired using a AIST-NT scanning probe microscope in intermittent contact mode. Commercially available Si cantilevers were used for the AFM measurements.

\section{Results and Discussion}

\section{Resonant Raman induced Fröhlich interaction in InSe}

Figure 1a presents a sample sketch with metal nanotriangles (NTs) on top of InSe flakes. Here, we used $\mathrm{Ag}, \mathrm{Au}$, and $\mathrm{Al}$ for the coupling between InSe and the plasmon of the metal NTs. Hexagonal arrays of the metal NTs have localized surface plasmon resonances (LSPR) in the visible range, which can be tuned via changing the material, size, shape, or dielectric environment ${ }^{26,30}$.

A typical SEM image of the sample after NSL using Ag is shown in Figure 1b. As can be seen, Ag formed nanotriangles homogeneously covering InSe without damaging the flake. Figure $1 \mathrm{c}-\mathrm{f}$ are the optical images and AFM topographies of one of the InSe samples with different layer thicknesses on a $300 \mathrm{~nm} \mathrm{SiO}_{2} / \mathrm{Si}$ substrate. Like other layered semiconductors, InSe has a wellknown layer-dependent optical bandgap ${ }^{18,31}$. We thus determined the InSe layer number using the PL peak position. The details of thickness determination via PL peak position are presented in the supplementary information, SI1. The PL spectrum of $7 \mathrm{~L} \mathrm{InSe} \mathrm{is} \mathrm{shown} \mathrm{in} \mathrm{the} \mathrm{inset} \mathrm{of} \mathrm{Figure}$ 1e. Using Voigt fitting the PL peak is determined to be centred at $(1.35 \pm 0.01) \mathrm{eV}$ corresponding to $7 \mathrm{~L} \mathrm{InSe} \mathrm{in} \mathrm{good} \mathrm{agreement} \mathrm{with} \mathrm{the} \mathrm{literature}{ }^{31}$. Before turning our attention to the plasmonic hot electron induced Fröhlich interaction, we investigated non-resonant and near-resonant Raman scattering spectra of InSe. This helps in the evaluation of polar modes in InSe since, at 

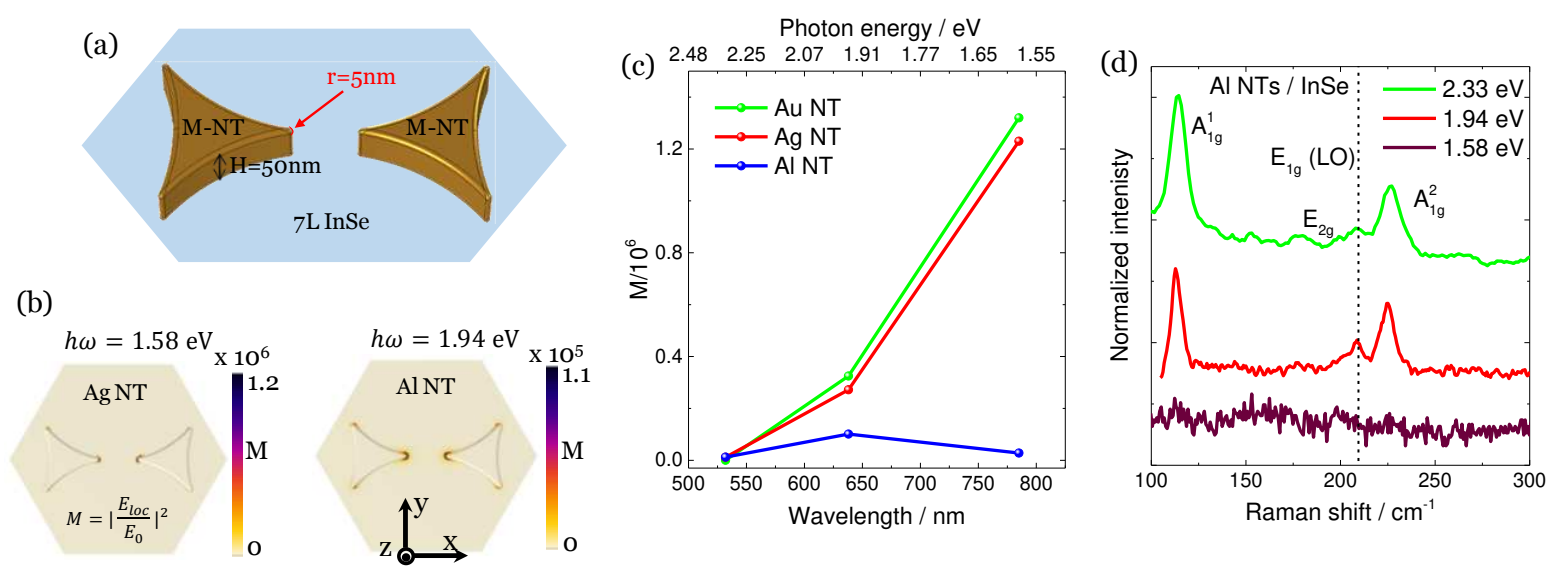

Figure 2 (a) Sketch of the model used in the FEM simulation of InSe/metal NTs. (b) Simulated electric field intensity $M$ distribution at two different wavelengths for Ag and Al NTs. Simulated transmittance spectra of InSe/Ag, Au, and Al NTs systems for the spectral range from 380 to $1200 \mathrm{~nm}$ together with $M$ distribution for Au NTs at $785 \mathrm{~nm}$ excitation are presented in SI3. (c) Absolute value of calculated maximum $M$ at three different wavelengths. A similar plasmonic behaviour is expected for Ag and Au NTs on InSe for three selected wavelengths while InSe with Al NTs shows a maximum at $638 \mathrm{~nm}$ excitation. (d) Raman spectra of 7L InSe with Al NTs acquired under three different wavelengths. As expected from simulations we can resolve the polar modes of InSe best at $638 \mathrm{~nm}(1.94 \mathrm{eV})$ excitation. Raman spectra of InSe with Au NTs are presented in SI3.

resonance excitation, Raman scattering is dominated by the exciton-LO phonon Fröhlich interaction ${ }^{21}$.

Figure 1 g displays both non-resonant and resonant Raman spectra of $7 \mathrm{~L}$ InSe measured at $1.94 \mathrm{eV}$ and $2.41 \mathrm{eV}$ excitation, respectively. In the non-resonant Raman spectrum, we observed three prominent first order Raman modes around $114 \mathrm{~cm}^{-1}, 178 \mathrm{~cm}^{-1}$, and $225 \mathrm{~cm}^{-1}$ assigned to $\mathrm{A}_{1 \mathrm{~g}}$, $\mathrm{E}_{2 \mathrm{~g}}$, and $\mathrm{A}^{2}{ }_{1 \mathrm{~g}}$ modes, respectively, (see Figure $\mathrm{h}$ ) according to the irreducible representation of the symmetry group $C_{3 v}^{5}\left(\gamma\right.$-InSe) ${ }^{32}$. There is another in-plane $\mathrm{E}_{2 \mathrm{~g}}$ mode around $40 \mathrm{~cm}^{-1}$ for InSe, which is inaccessible in our experiment. Additionally, we can also observe a very weak contribution of the polar $\mathrm{A}_{1}$ (LO) around $200 \mathrm{~cm}^{-1}$, while the increasing background around 300 $\mathrm{cm}^{-1}$ is the contribution from $\mathrm{Si} 2^{\text {nd }}$ order phonon scattering. Interestingly, under near-resonant Raman condition when excited by the $2.41 \mathrm{eV}$ laser, the energy of which is close to the $\mathrm{E}_{1}$ gap (around $2.42 \mathrm{eV}$ ) of InSe, together with the non-polar Raman modes, we observed a dramatic enhancement of the polar modes (asymmetric feature around $200 \mathrm{~cm}^{-1}$ ). The peak centred at 200 $\mathrm{cm}^{-1}$ can be assigned to $\mathrm{A}_{1}(\mathrm{LO})$ and the shoulder on the higher frequency side around $210 \mathrm{~cm}^{-1}$ to E (LO), both of them are more active in the infrared absorption than in the ordinary Raman spectra 20,33,34. However, under near-resonant Raman excitation $q$-dependent scattering 
dominates and dictates the Raman symmetry selection rules ${ }^{35}$. Therefore, infrared active polar phonons are enhanced in the Raman spectra of InSe with strong $A_{1}$ (LO) and weaker E (LO) contributions in backscattering geometry.

\section{Plasmonic hot electron induced Fröhlich interaction in InSe}

Figure 1i shows the Raman spectra of $7 \mathrm{~L} \mathrm{InSe}\left(\mathrm{E}_{\mathrm{g}}=1.35 \mathrm{eV}\right)$ without and with Ag NTs. While the Raman spectra of InSe without Ag NTs show first order Raman modes at $2.33 \mathrm{eV}$ and $1.94 \mathrm{eV}$ excitations, no Raman modes are observed under $1.58 \mathrm{eV}$ excitation. This could be due to the excitation energy, which is far away from the $\mathrm{E}_{1}$ and the Raman scattering is less efficient near weakly allowed $\mathrm{E}_{g}$. In contrast, the Raman spectra of InSe/Ag NTs system exhibit significant modifications. First, we observed the strongest SERS enhancement at $1.58 \mathrm{eV}$ excitation. This is unexpected taking our recent work into account since we showed that hexagonally packed Ag NTs of the same geometry on a $\mathrm{SiO}_{2} / \mathrm{Si}$ substrate have a LSPR around $1.9 \mathrm{eV}$ and are thus resonant with $1.94 \mathrm{eV}$ excitation ${ }^{26}$. However, the present investigation clearly shows that the $1.58 \mathrm{eV}$ excitation is closer to resonance if not at resonance with LSPR of Ag NTs suggesting a shift of the LSPR to lower energies due to the change of dielectric environment in the presence of few layer InSe at the bottom ${ }^{30}$. Thanks to the resonant SERS effect, together with first order Raman modes we can now observe the polar modes in InSe at $1.58 \mathrm{eV}$ excitation. The broad feature between 150 $\mathrm{cm}^{-1}$ and $190 \mathrm{~cm}^{-1}$ is the combination of in-plane $\mathrm{E}_{2 \mathrm{~g}}$ mode of InSe and the contribution from $\mathrm{In}_{2} \mathrm{O}_{3}$ (a byproduct of oxidation in ultrathin InSe ) 36,37 .

To understand the role of InSe on the LSPR of metal NTs, we performed FEM simulations using COMSOL multiphysics 5.6 in 3D platform. Figure 2a presents the model used to simulate the experimental geometry studied in this work. Details of the $3 \mathrm{D}$ model can be found in the experimental section and SI2. The transmittance spectra of the three metal NTs (Au, Ag, Al) on $7 \mathrm{~L}$ of InSe computed in the spectral range from $360 \mathrm{~nm}(3.44 \mathrm{eV})$ to $1200 \mathrm{~nm}(1.03 \mathrm{eV})$ are presented in Figure SI2a. The distribution of the electric field intensity enhancement $M$ around the $\mathrm{Ag}$ and $\mathrm{Al} \mathrm{NTs}$ calculated at $1.58 \mathrm{eV}$ and $1.94 \mathrm{eV}$ excitations, respectively, are shown in Figure 2b. The $M$ distribution for Au NTs at $1.58 \mathrm{eV}$ excitation can be found in Figure SI2b. As expected, the corners of the NTs generate the strongest electric field enhancement in agreement to previous works ${ }^{26,38}$. The absolute values of the calculated maximum $M$ for Ag, Au, and Al NTs at three different wavelengths are displayed in Figure 2c. From Figure 2c and Figure SI2a, we can see that $\mathrm{Au}$ and Ag NTs show strongest enhancement under $1.58 \mathrm{eV}$ excitation compared to the other two energies. Therefore, excitation with $1.58 \mathrm{eV}$ shall produce stronger plasmonic field and generate highly energetic hot electrons in both metal NTs. This result thus confirms that the strongest enhancement in the Raman spectra of InSe with Ag or Au NTs should appear at $1.58 \mathrm{eV}$ excitation 
(Raman spectrum of InSe/Au NTs at $1.58 \mathrm{eV}$ excitation is presented in Figure SI2c). Under this excitation condition the two polar modes ( $\mathrm{A}_{1}(\mathrm{LO})$ and $\mathrm{E}(\mathrm{LO})$ ) are observed in the Raman spectra.

From the simulation results shown in Figure $2 \mathrm{c}$ and SI2a it is clear that the LSPR of Al NTs shifts towards $638 \mathrm{~nm}(1.94 \mathrm{eV})$ and one should expect the strongest plasmonic enhancement effect at $1.94 \mathrm{eV}$ excitation. Figure 2d presents Raman spectra of InSe with Al NTs acquired at all three excitation wavelengths. Indeed we observe the strongest plasmonic enhancement at $1.94 \mathrm{eV}$ excitation in terms of the appearance of the two polar modes. No Raman features are visible at $1.58 \mathrm{eV}$ since this excitation energy is non-resonant for both the InSe and the LSPR of Al NTs. Please note that the Raman spectra of InSe at $2.33 \mathrm{eV}$ excitation is the combination of plasmonic enhancement and near-resonant Raman effect as discussed above.

One notable difference between the near-resonant Raman spectra in Figure $1 \mathrm{~g}$ and the SERS spectra at $1.58 \mathrm{eV}$ excitation in Figure $1 \mathrm{i}$ is the relative intensity ratio between $\mathrm{A}_{1}$ (LO) and $\mathrm{E}$ (LO). In the case of near-resonant Raman excitation, the $A_{1}$ (LO) mode appears much stronger compared to the $\mathrm{E}$ (LO) one in agreement with literature ${ }^{20,39,40}$. On the contrary, the $\mathrm{E}$ (LO) mode is more enhanced than the $A_{1}$ (LO) mode in resonant SERS spectra, in which the probability of generated hot electron in NTs collected by InSe is the maximum, consistently in the present study. This is likely due to hot carrier induced EPC of the E (LO) polar mode being much stronger in InSe as suggested in a recent DFT investigation ${ }^{41}$. Additionally, our simulation shows that the stronger in-plane plasmonic field component at $1.58 \mathrm{eV}$ excitation (see Figure SI2d) couples to the in-plane $\mathrm{E}(\mathrm{LO})$ mode better than $\mathrm{A}_{1}(\mathrm{LO})$ mode resulting in enhanced relative intensity.

The Raman spectra presented in Figure $1 \mathrm{i}$ and $2 \mathrm{~d}$ clearly indicate that the plasmonic coupling between InSe and metal NTs is the sole contributor to the observation of the polar modes in the Raman spectrum excited by $1.58 \mathrm{eV}$ and $1.94 \mathrm{eV}$, which would be non-resonant without NTs. There are two possible plasmonic phenomena, which can contribute to the observation of the polar modes: i) the local field strongly confined at the corners of NTs as shown in Figure $2 b$ and SI2d and ii) doping by the plasmonic hot electrons. A similar behaviour was also observed for the case of a monolayer $\mathrm{MoS}_{2} / \mathrm{Au}$ nanodisc system in our previous work ${ }^{42}$. The strongly confined plasmonic field in a monolayer $\mathrm{MoS}_{2}$ sandwiched by a nanodisc and a sharp metallic tip generated hot electrons, which excite an infrared active $\mathrm{A}_{2 \mathrm{u}}$ mode under $1.58 \mathrm{eV}$ excitation energy, far below the bandgap of the monolayer $\mathrm{MoS}_{2}$.

To test our hypothesis, we partially encapsulated InSe by a few layers of hBN ( 10 L) and deposited Ag NTs using the same procedure. The plasmonic field decays exponentially through 

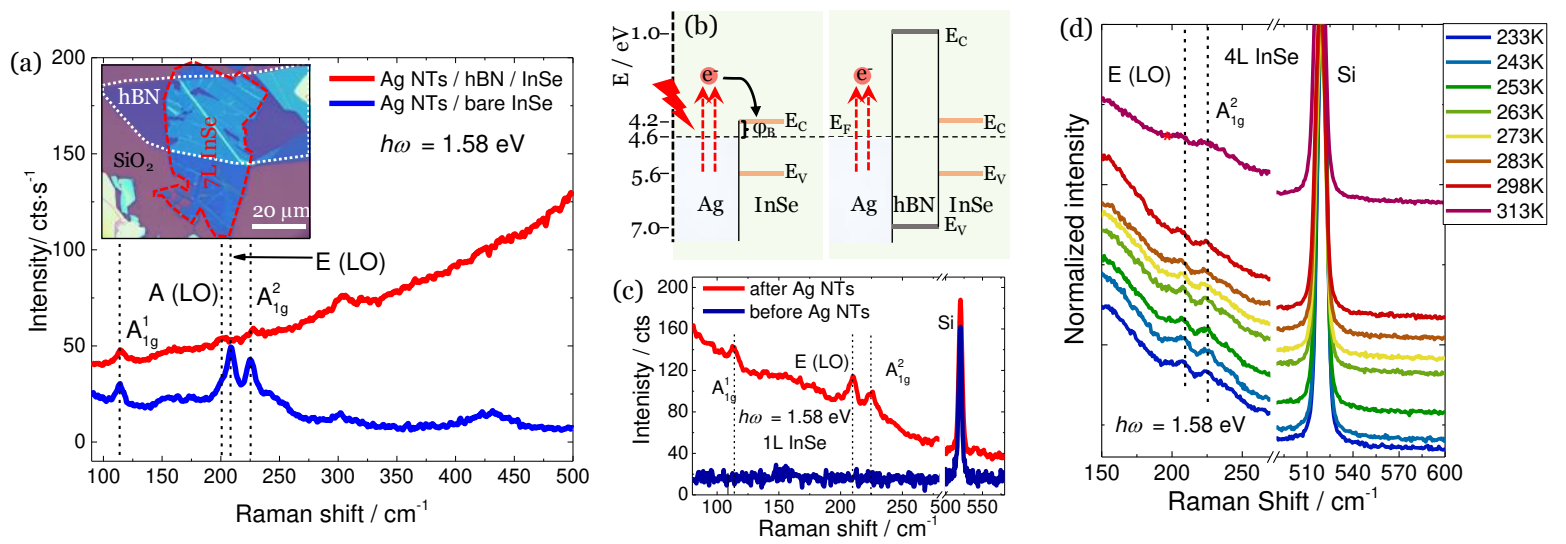

Figure 3. (a) Raman spectra taken with $1.58 \mathrm{eV}$ excitation of hBN encapsulated and bare InSe/Ag NTs demonstrating the influence of plasmonic hot electron doping. The inset is the optical image of the partially encapsulated sample used for the measurements. The areas marked by the white dotted and red dashed lines indicate hBN and InSe, respectively. (b) Simplified diagram of the band alignment illustrating the hot electron doping process. Left panel: InSe with Ag NTs, right panel: InSe/hBN/Ag NTs. The values are taken from the refs..$^{13,43,44}$ (c) Raman spectra of $1 \mathrm{~L}$ InSe before and after Ag NTs deposition. The bandgap of 1L InSe is well above the excitation energy at $1.58 \mathrm{eV}$. (d) Temperature dependent Raman measurements of 4L InSe/Ag NTs at $1.58 \mathrm{eV}$ excitation. The acquisition parameters of the Raman measurements are $30 \mathrm{~s} \times 2$ (7L) and $150 \sec \times 2(1 \mathrm{~L}), 10 \mu \mathrm{W}$ under $100 \mathrm{x}, 0.9 \mathrm{NA}$ objective. The acquisition parameters for the temperature dependent Raman measurements were $150 \sec x 2,10 \mu \mathrm{W}$ under 50x, 0.45 NA objective.

the hBN capping layer with a decay constant (see Figure SI3) and hBN blocks InSe from being doped by plasmonic hot electrons. Therefore, we are able to determine the influence of the two effects mentioned above on hBN capped InSe directly.

Figure 3a displays an optical image of the heterostructure showing bare and hBN encapsulated InSe regions together with two representative Raman spectra of the two regions after Ag NTs deposition using $1.58 \mathrm{eV}$ excitation. The Raman spectra of bare InSe in the presence of Ag NTs exhibits a strong contribution of the polar modes as well as enhancement of the nonpolar modes in agreement with the results shown in Figure 1i. However, the intensities of the nonpolar first order Raman modes display weaker enhancement for hBN encapsulated InSe with Ag NTs than without Ag NTs, since we did not observe any Raman features in the absence of Ag NTs at $1.58 \mathrm{eV}$ excitation as demonstrated in Figure 1i. The weak enhancement of the non-polar Raman modes can be due to the screening of the plasmonic field by the $\mathrm{hBN}$ interlayer. The effect of this weak plasmonic coupling can also be observed through the enhanced PL (by a factor of 5.4) from hBN encapsulated InSe after Ag NTs deposition (see Figure SI3), which also leads to an increased background in the red spectrum in Figure 3 a. 
For bare InSe with Ag NTs, on the other hand, we did not observe any PL. This is likely to be due to the efficient exciton dissociation known to occur at the semiconductor/metal interface ${ }^{40}$. However, the hBN capping blocks the photoexcited charge transfer from InSe to the Ag NTs. Therefore, we can observe the PL enhancement via plasmonic coupling. The Raman spectra taken from the hBN encapsulated InSe with Ag NTs show negligible contribution from the polar modes, especially the E (LO) mode. This is a stark contrast to the non-polar Raman modes in hBN capped InSe. The appearance of a much weaker $\mathrm{A}_{1}$ (LO) mode in $\mathrm{hBN}$ capped InSe indicates that the contribution of local plasmonic field gradient induced selection rules (as discussed above) may aid to the weak appearance of $\mathrm{A}_{1}$ (LO) mode. On the other hand, the vanishing $\mathrm{E}$ (LO) mode suggests that the plasmonic hot electron doping plays the critical role in activating the polar modes in non-resonant Raman spectra for InSe.

Figure $3 \mathrm{~b}$ depicts an approximated band alignment diagram for the heterostructures shown in Figure $3 a$ indicating the plasmonic hot electron doping mechanism in InSe. The values needed to construct the band alignment were taken from the literature 13,43,44. After excitation at LSPR, the plasmon can decay radiatively or non-radiatively by transferring the energy to the conduction electrons in the metal nanostructures. This process creates highly energetic hot electrons, which can escape from the metal and be collected by a semiconductor in contact with the plasmonic structures. The energy transferred to the hot electrons from the noble metal nanostructures at LSPR excitation is higher than $1 \mathrm{eV}$ and can reach up to $4 \mathrm{eV}{ }^{11}$. Therefore, hot electrons generated in Ag NTs can easily overcome the barrier height $\varphi_{B}$ (approximately $0.4 \mathrm{eV}$ ) and dope InSe. When encapsulated by hBN, this wide bandgap insulator creates a blocking layer for the hot electrons to be collected by InSe (right panel in Figure 3b). As a result, polar modes in InSe are weak in the red spectrum presented of Figure $3 \mathrm{a}$.

One of the critical sources of the Fröhlich interaction in polar semiconductors is the intraband EPC stemming from the scattering of charges within the same valence or conduction bands by LO phonons. Plasmonic hot electron doping in InSe meets such criteria since electrons escaping the metal nanostructures are collected by the conduction band of InSe. These in turn interact with the long-range macroscopic polarization density associated with either the creation or annihilation of LO phonons in InSe 7. The charge transport driven by an electric field through InSe can also be influenced by such a macroscopic field, which consequently reduces the mobility.

Even though the electronic transition at the bandgap in InSe is weak at the normal incidence, it can still induce resonant Raman scattering in a strong plasmonic field. Hence, the strong intensity of the polar phonons observed in $7 \mathrm{~L}$ InSe may also be activated via this resonance 

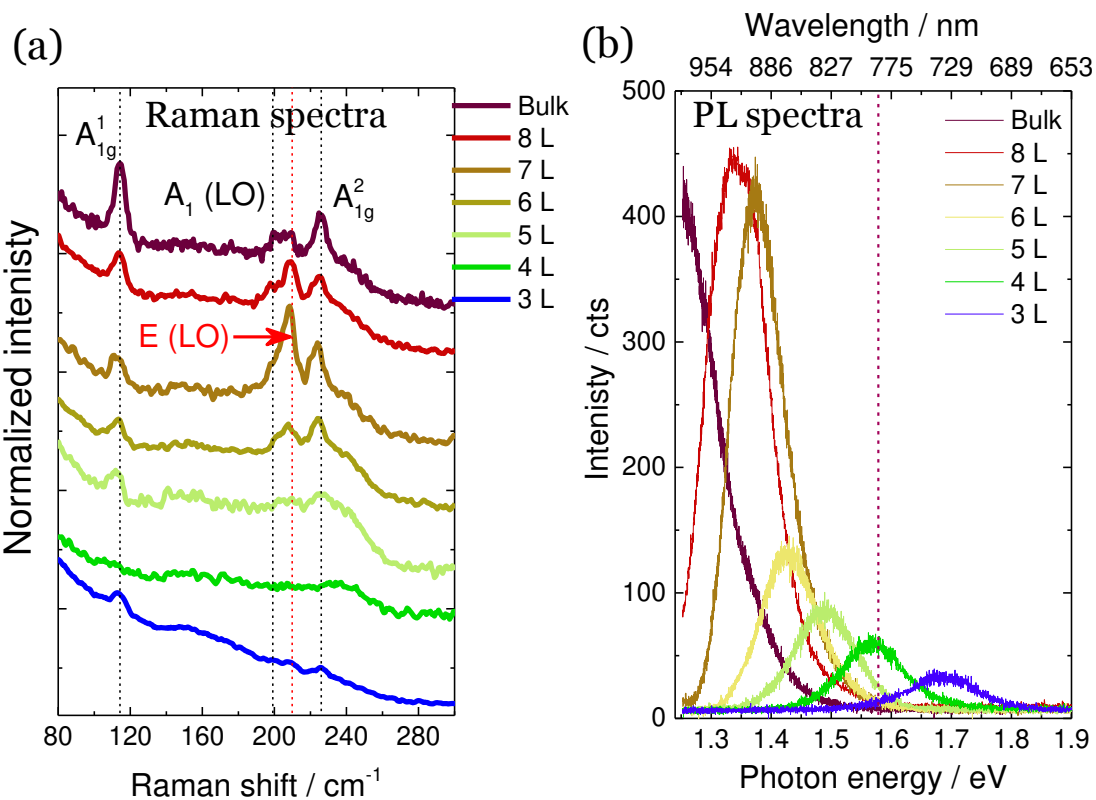

Figure 4. Layer dependent (a) Raman excited at $1.58 \mathrm{eV}$ and (b) corresponding PL spectra of InSe. There is a drastic drop of intensity of the polar LO modes from 7L to 6L corresponding to the thickness where the direct to indirect bandgap transition of InSe occurs.

phenomenon. To examine this scenario, we prepared a 1L InSe with Ag NTs sample. The PL spectra of the flake confirming the layer thickness is presented in Figure SI4. The bandgap of $1 \mathrm{~L}$ InSe at $\mathrm{RT}$ is $2.1 \mathrm{eV}$, i.e. $1.58 \mathrm{eV}$ excitation is well below the electronic transition. Therefore, if the appearance of the polar phonons would only stem from the coupling between these phonons and the weakly allowed $E_{g}$ transition in a strong plasmonic field, we should not observe them for $1 \mathrm{~L}$ InSe. Instead, the polar phonons in 1L InSe when covered with Ag NTs are clearly observed (Figure 3c).

Furthermore, by conducting a temperature dependent Raman investigation on two selected thicknesses (4L: $\mathrm{E}_{\mathrm{g}} \geq 1.58 \mathrm{eV}$ and $7 \mathrm{~L}: \mathrm{E}_{\mathrm{g}}<1.58 \mathrm{eV}$ at RT) of InSe with Ag NTs at $1.58 \mathrm{eV}$ excitation, it becomes clear that with decreasing temperature the bandgap of $4 \mathrm{~L}(7 \mathrm{~L})$ InSe moves away (towards) from the excitation energy of $1.58 \mathrm{eV}$, i.e. the resonance condition is tuned correspondingly. The result for $4 \mathrm{~L}$ InSe is presented in Figure $3 \mathrm{~d}$ and for $7 \mathrm{~L}$ InSe in Figure SI5. As depicted in Figure 3d, Raman signature of polar E (LO) and non-polar $\mathrm{A}^{2}{ }_{1 \mathrm{~g}}$ phonons in $4 \mathrm{~L}$ InSe are visible in the spectra and their intensities remain constant throughout the temperature range $(233-313) \mathrm{K}$. Due to strong increasing Rayleigh background the $\mathrm{A}^{1}{ }_{1 \mathrm{~g}}$ mode around $114 \mathrm{~cm}^{-1}$ could not be observed under 50x, 0.45 NA objective used for the temperature dependent study. The constant intensity of the $\mathrm{E}$ (LO) mode over the whole temperature range indicates that the activation of polar phonons in InSe at $1.58 \mathrm{eV}$ excitation does not depend on $\mathrm{E}_{\mathrm{g}}$ transition. 
On the other hand, the temperature dependent Raman spectra of $7 \mathrm{~L} \mathrm{InSe} / \mathrm{Ag}$ NTs at $1.58 \mathrm{eV}$ excitation presented in Figure SI5 reveal that the intensity of polar phonons is enhanced when the temperature-dependent bandgap approaches the excitation energy at lower temperature. This is because with decreasing temperature the bandgap of $7 \mathrm{~L}$ InSe approaches the excitation energy of $1.58 \mathrm{eV}$ meaning resonance with $\mathrm{E}_{\mathrm{g}}$ is established. This phenomenon suggests that the fundamental bandgap $\mathrm{E}_{\mathrm{g}}$ plays a role in enhancing the polar LO modes. However, as we showed in this work hot electron doping is the prerequisite for observing the polar LO modes, thus Fröhlich interaction in InSe takes place.

\section{Layer dependent evolution of Fröhlich interaction in InSe}

Figure 4 depicts the layer dependent Raman spectra of InSe with Ag NTs with corresponding PL spectra acquired prior to NTs deposition. As can be seen, the intensity of the polar modes (especially the E (LO) mode) gradually increases from bulk to $7 \mathrm{~L}$ of InSe, where it reaches a maximum. This is likely due to the resonant condition since, with decreasing layer number the bandgap of InSe approaches the excitation energy of $1.58 \mathrm{eV}$. After that, there is a drastic drop of intensity of $\mathrm{E}$ (LO) mode at $6 \mathrm{~L}$ InSe, which then continues to decrease gradually with further decreasing layer number. The corresponding PL spectra in Figure $4 \mathrm{~b}$ clearly reveals that the transition from direct to indirect bandgap at RT also occurs from $7 \mathrm{~L}$ to $6 \mathrm{~L}$ InSe. Details of the layer dependent bandgap evolution in InSe are discussed in SI1.

In a polar semiconductor, free carrier plasmons couple to LO phonons via the interaction between the macroscopic electric field associated with LO phonons and the electric field induced by the free carriers. The strength of this coupling depends on the nature of the bandgap since conduction-electron plasmons couple strongly to the LO phonon in a direct bandgap semiconductor due to the formation of an electron gas with small effective mass and large dielectric constant ${ }^{15}$. This is why a sudden drop of $\mathrm{E}(\mathrm{LO})$ phonon intensity from direct bandgap $7 \mathrm{~L}$ InSe to indirect bandgap 6L InSe is observed.

Since further thinning of InSe gradually increases the indirect bandgap and the energy difference between the indirect to direct transition in InSe, there is further gradual decrease of the LO phonons in layer dependent Raman spectra. Note that, the bandgap is increasing with decreasing the layer number in InSe, which also increases the barrier height at the InSe/Ag interface. This may also influence the doping probability. However, considering the fact that the hot electrons can gain large energy in Ag nanostructures ${ }^{11}$, a barrier height of $<1 \mathrm{eV}$ to InSe can easily be overcome. Additionally, the LSPR of Ag NTs shall move to higher energy with decreasing 
thickness of InSe, especially for very thin flakes, resulting in different coupling between InSe and Ag NTs.

To understand how the LSPR compares at different thicknesses we calculated the local electric field enhancement due to $\mathrm{Ag}$ NTs for $2 \mathrm{~nm}$ InSe (representing 2L InSe) at $1.58 \mathrm{eV}$ excitation energy. The simulation results are presented in Figure SI6. The local electric field enhancement of Ag NTs on 2L InSe and 7L InSe are comparable, i.e. the sharp intensity decrease of polar phonons at ultrathin flakes cannot be explained by a change of the LSPR of Ag NTs. Therefore, the decreasing intensity of the polar phonons below 6L InSe is due to the reduced Fröhlich coupling as is clearly indicated in Figure 4a.

This is a very important result and critical since the Fröhlich interaction strongly suppresses the mobility of the charge carriers thus reducing any device performance. The reported sequence of procedure, experimental results, simulations and cross referenced interpretations provide a clear picture of layer-dependent Fröhlich interaction mechanism occurring in InSe, which are relevant in future device applications of this promising 2D material.

\section{Conclusion}

We studied the Fröhlich interaction in InSe by monitoring the strength of polar LO modes induced by plasmonic hot electron doping. We deposited three metal ( $\mathrm{Al}, \mathrm{Ag}$, and $\mathrm{Au}$ ) nanostructures on InSe and excited the heterostructure system near LSPR. The laser lines (1.58 $\mathrm{eV}$ and $1.94 \mathrm{eV}$ ) used in this work are far away from the electronic transition energy at $\mathrm{E}_{1}$, i.e. far away from the resonant-Raman condition for InSe. We observed the strong appearance of polar LO modes of InSe in resonant SERS condition, which are otherwise very weak in ordinary Raman conditions. The combination of the strong local field at the corners of the NTs and the hot electrons generated due to near LSPR excitation activates and enhances these polar phonon modes enabling their observation in the Raman measurements. We performed FEM simulations to calculate the plasmonic coupling between InSe and metal NTs. We observed an excellent agreement between the simulation and experiments showing that the polar LO modes are only observed when the excitation energy approaches the LSPR (the condition for the largest concentration of hot electrons excited in the metal nanostructures). To elucidate our findings further, we conducted a series of experiments including a repetition of the SERS experiments on hBN encapsulated InSe, which confirmed that the plasmonic hot electron doping is the origin of the polar modes appearing in the spectra. Finally, we investigated the layer-dependent Fröhlich interaction in InSe. We found that intensity of polar modes initially increases with decreasing layer number indicating increasing Fröhlich coupling. The sudden decrease of intensity of the 
polar modes from $7 \mathrm{~L}$ to $6 \mathrm{~L}$ coincides with the transition from direct to indirect bandgap at RT. The polar mode intensities gradually decrease with further decreasing layer number. The reduction of the intensity of the polar modes suggests that the Fröhlich coupling is smaller at thinner flakes below $7 \mathrm{~L}$ due to the indirect bandgap nature. Our results provide the first experimental picture of layer-dependent Fröhlich coupling in InSe, which is vital for any potential device application of this promising material.

\section{Acknowledgment}

We acknowledge funding by the German Science Fundation DFG via DFG ZA 146/44-1, and the SMWK/ESF (RL ESF Hochschule und Forschung 2014-433 2020 mit Antragsnummer 100380093) projects for supporting the research work. We also thank Ms. Doreen Dentel of solid surfaces analysis group in Chemnitz for the SEM measurements.

\section{Author Contributions}

M.R. and M.A.A. performed the measurements and analysis the date. M.R. and D.R.T.Z. involved in the evaluation and interpretation of the results. M.R., L.H., and T.M. developed the simulation model and performed the calculations. M.R. wrote the manuscript. All authors contributed to the understanding of the experimental findings and writing the manuscript.

Corresponding author: All the correspondence and the material requests should be addressed to Mahfujur Rahaman, mahfujur.rahaman@physik.tu-chemnitz.de.

\section{Competing interest}

Authors declare no competing interest for this manuscript.

\section{Data availability}

All data generated and analyzed during this investigation are available from the corresponding author upon reasonable request.

\section{References}

1 Bandurin, D. A. et al. High electron mobility, quantum Hall effect and anomalous optical response in atomically thin InSe. Nature Nanotechnology 12, 223-227, doi:10.1038/nnano.2016.242 (2017).

2 Li, L. et al. Black phosphorus field-effect transistors. Nature Nanotechnology 9, 372-377, doi:10.1038/nnano.2014.35 (2014).

3 Island, J. O., Steele, G. A., Zant, H. S. J. v. d. \& Castellanos-Gomez, A. Environmental instability of few-layer black phosphorus. 2D Materials 2, 011002, doi:10.1088/2053-1583/2/1/011002 (2015).

4 Feng, W., Zheng, W., Gao, F. \& Hu, P. Atomically thin InSe: A high mobility two-dimensional material. Science China Technological Sciences 6o, 1121-1122, doi:10.1007/s11431-016-9004-X (2017). 
Feng, W., Zheng, W., Cao, W. \& Hu, P. Back Gated Multilayer InSe Transistors with Enhanced Carrier Mobilities via the Suppression of Carrier Scattering from a Dielectric Interface. Advanced Materials 26, 6587-6593, doi:https://doi.org/10.1002/adma.201402427 (2014). Fröhlich, H. Electrons in lattice fields. Advances in Physics doi:10.1080/00018735400101213 (1954). Ma, J., Xu, D., Hu, R. \& Luo, X. Examining two-dimensional Fröhlich model and enhancing the electron mobility of monolayer InSe by dielectric engineering. Journal of Applied Physics 128, 035107, doi:10.1063/5.0015102 (2020). Influence on the Transport and Optical Properties of Hole-Doped Single-Layer InSe. Physical Review Letters 123, 176401, doi:10.1103/PhysRevLett.123.176401 (2019).

Sohier, T., Calandra, M. \& Mauri, F. Two-dimensional Fröhlich interaction in transition-metal dichalcogenide monolayers: Theoretical modeling and first-principles calculations. Physical Review B 94, 085415, doi:10.1103/PhysRevB.94.085415 (2016). Li, W., Poncé, S. \& Giustino, F. Dimensional Crossover in the Carrier Mobility of Two-Dimensional Semiconductors: The Case of InSe. Nano Letters 19, 1774-1781, doi:10.1021/acs.nanolett.8bo4799 (2019).

Clavero, C. Plasmon-induced hot-electron generation at nanoparticle/metal-oxide interfaces for photovoltaic and photocatalytic devices. Nature Photonics 8, 95-103, doi:10.1038/nphoton.2013.238 (2014). $\mathrm{Hu}, \mathrm{S}$. et al. Au-InSe van der Waals Schottky junctions with ultralow reverse current and high photosensitivity. Nanoscale 12, 4094-4100, doi:10.1039/C9NRo8791E (2020). Sun, H., Wang, Z. \& Wang, Y. Band alignment of two-dimensional metal monochalcogenides MXs ( $\mathrm{M}=\mathrm{Ga}, \mathrm{In} ; \mathrm{X}=\mathrm{S}, \mathrm{Se}, \mathrm{Te})$. AIP Advances 7, 095120, doi:10.1063/1.4986385 (2017). Review 137, A1896-A1902, doi:10.1103/PhysRev.137.A1896 (1965). Physical Review B 33, 1222-1232, doi:10.1103/PhysRevB.33.1222 (1986).

Leisgang, N. et al. Optical second harmonic generation in encapsulated single-layer InSe. AIP Advances 8, 105120, doi:10.1063/1.5052417 (2018).

Hao, Q. et al. Phase Identification and Strong Second Harmonic Generation in Pure $\varepsilon$-InSe and Its Alloys. Nano Letters 19, 2634-2640, doi:10.1021/acs.nanolett.9bo0487 (2019).

Brotons-Gisbert, M. et al. Nanotexturing To Enhance Photoluminescent Response of Atomically Thin Indium Selenide with Highly Tunable Band Gap. Nano Letters 16, 3221-3229, doi:10.1021/acs.nanolett.6boo689 (2016).

Mudd, G. W. et al. The direct-to-indirect band gap crossover in two-dimensional van der Waals Indium Selenide crystals. Scientific Reports 6, 39619, doi:10.1038/srep39619 (2016).

Sánchez-Royo, J. F. et al. Electronic structure, optical properties, and lattice dynamics in atomically thin indium selenide flakes. Nano Research 7, 1556-1568, doi:10.1007/s12274-014-0516-x (2014). Ashokan, S., Jain, K. P., Balkanski, M. \& Julien, C. Resonant Raman scattering in quasi-twodimensional InSe near the $\mathrm{M}_{0}$ and $\mathrm{M}_{1}$ critical points. Physical Review $B$ 44, 11133-11142, doi:10.1103/PhysRevB.44.11133 (1991).

Hamer, M. J. et al. Indirect to Direct Gap Crossover in Two-Dimensional InSe Revealed by AngleResolved Photoemission Spectroscopy. ACS Nano 13, 2136-2142, doi:10.1021/acsnano.8bo8726 (2019).

Atwater, H. A. \& Polman, A. Plasmonics for improved photovoltaic devices. Nature Materials 9, 205-213, doi:10.1038/nmat2629 (2010).

Mukherjee, S. et al. Hot Electrons Do the Impossible: Plasmon-Induced Dissociation of H2 on Au. Nano Letters 13, 240-247, doi:10.1021/nl303940z (2013).

Castellanos-Gomez, A. et al. Deterministic transfer of two-dimensional materials by all-dry viscoelastic stamping. 2D Materials 1, 011002, doi:10.1088/2053-1583/1/1/011002 (2014).

Rahaman, M., Moras, S., He, L., Madeira, T. I. \& Zahn, D. R. T. Fine-tuning of localized surface plasmon resonance of metal nanostructures from near-Infrared to blue prepared by nanosphere lithography. Journal of Applied Physics 128, 233104, doi:10.1063/5.0027139 (2020). Adachi, S. The Handbook on Optical Constants of Semiconductors. (WORLD SCIENTIFIC, 2012). Johnson, P. B. \& Christy, R. W. Optical Constants of the Noble Metals. Physical Review B 6, 43704379, doi:10.1103/PhysRevB.6.4370 (1972). 
McPeak, K. M. et al. Plasmonic Films Can Easily Be Better: Rules and Recipes. ACS Photonics 2, 326-333, doi:10.1021/ph5004237 (2015).

30 Duval Malinsky, M., Kelly, K. L., Schatz, G. C. \& Van Duyne, R. P. Nanosphere Lithography: Effect of Substrate on the Localized Surface Plasmon Resonance Spectrum of Silver Nanoparticles. The Journal of Physical Chemistry B 105, 2343-2350, doi:10.1021/jp002906x (2001).

Song, C. et al. Largely Tunable Band Structures of Few-Layer InSe by Uniaxial Strain. ACS Applied Materials \& Interfaces 10, 3994-4000, doi:10.1021/acsami.7b17247 (2018).

32 Tamalampudi, S. R. et al. Thickness-Dependent Resonant Raman and E' Photoluminescence Spectra of Indium Selenide and Indium Selenide/Graphene Heterostructures. The Journal of Physical Chemistry C 123, 15345-15353, doi:10.1021/acs.jpcc.9b03457 (2019).

33 Gasanly, N. M., Yavadov, B. M., Tagirov, V. I. \& Vinogradov, E. A. Infrared and Raman Spectra of Layer InSe Single Crystals. physica status solidi (b) 89, K43-K48, doi:https://doi.org/10.1002/pssb.2220890155 (1978).

34 Kuroda, N. \& Nishina, Y. Resonant Raman scattering at higher Mo exciton edge in layer compound InSe. Solid State Communications 28, 439-443, doi:https://doi.org/10.1016/00381098(78)90834-7 (1978).

35 Martin, R. M. Theory of the One-Phonon Resonance Raman Effect. Physical Review B 4, 36763685, doi:10.1103/PhysRevB.4.3676 (1971).

36 Liu, D. et al. Large-scale synthesis of hexagonal corundum-type In2O3 by ball milling with enhanced lithium storage capabilities. Journal of Materials Chemistry A 1, 5274-5278, doi:10.1039/C3TAo0182B (2013).

37 Shi, L. et al. Oxidation Mechanism and Protection Strategy of Ultrathin Indium Selenide: Insight from Theory. The Journal of Physical Chemistry Letters 8, 4368-4373, doi:10.1021/acs.jpclett.7bo2059 (2017).

38 Murray, W. A., Suckling, J. R. \& Barnes, W. L. Overlayers on Silver Nanotriangles: Field Confinement and Spectral Position of Localized Surface Plasmon Resonances. Nano Letters 6, 1772-1777, doi:10.1021/nlo6o812e (2006).

39 Molas, M. R. et al. Raman spectroscopy of GaSe and InSe post-transition metal chalcogenides layers. Faraday Discussions, doi:10.1039/DoFDoooo7H (2021).

40 Lei, S. et al. Evolution of the Electronic Band Structure and Efficient Photo-Detection in Atomic Layers of InSe. ACS Nano 8, 1263-1272, doi:10.1021/nn405036u (2014).

41 Chen, Z. et al. Ultrafast dynamics of hot carriers in a quasi-two-dimensional electron gas on InSe. Proceedings of the National Academy of Sciences 117, 21962, doi:10.1073/pnas.2008282117 (2020).

42 Milekhin, A. G. et al. Giant gap-plasmon tip-enhanced Raman scattering of MoS2 monolayers on Au nanocluster arrays. Nanoscale 10, 2755-2763, doi:10.1039/C7NRo664oF (2018).

43 Schulman, D. S., Arnold, A. J. \& Das, S. Contact engineering for 2D materials and devices. Chemical Society Reviews 47, 3037-3058, doi:10.1039/C7CSo0828G (2018).

44 Wickramaratne, D., Weston, L. \& Van de Walle, C. G. Monolayer to Bulk Properties of Hexagonal Boron Nitride. The Journal of Physical Chemistry C 122, 25524-25529, doi:10.1021/acs.jpcc.8bo9o87 (2018). 


\section{Figures}
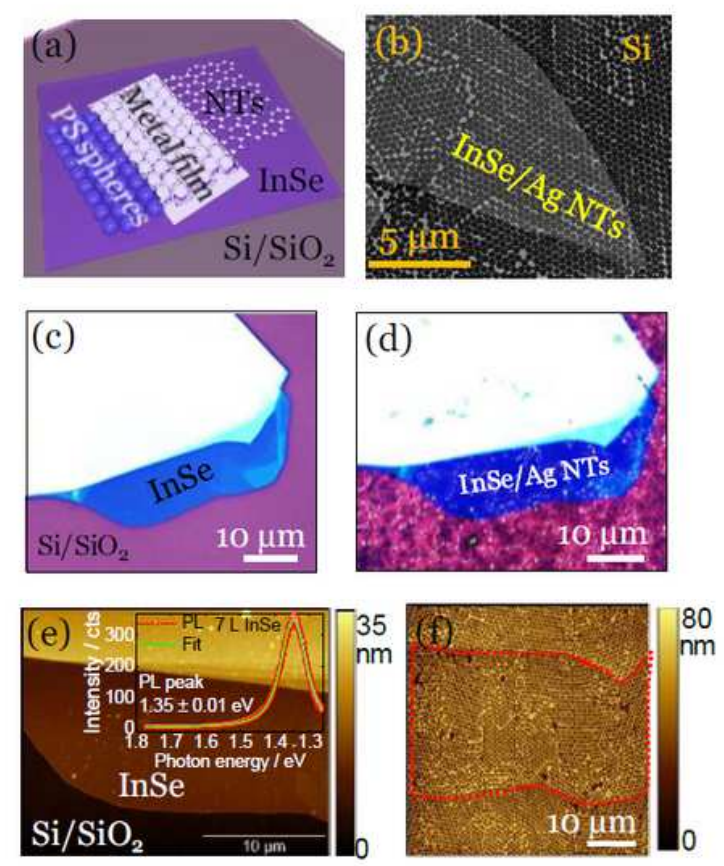
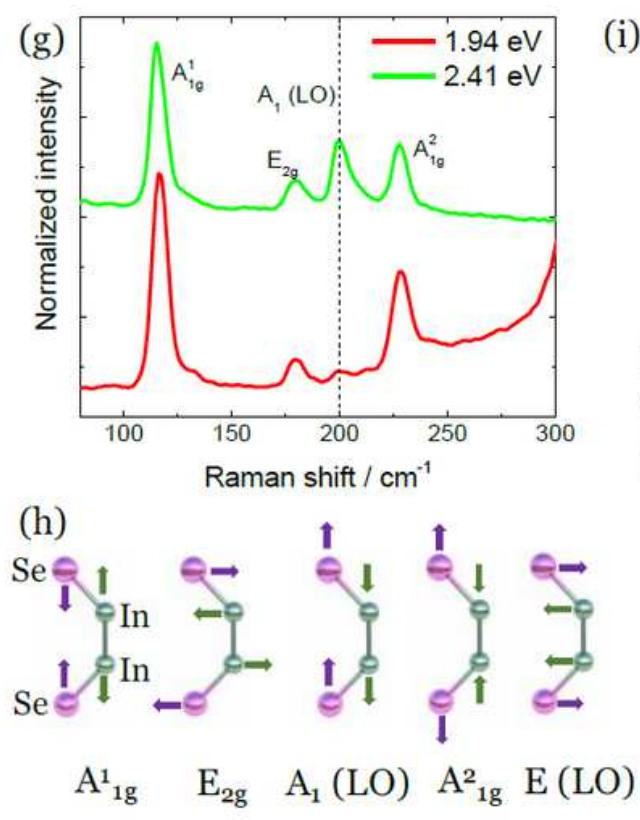

\section{Figure 1}

(a) Schematic representation of the sample preparation. Polystyrene spheres of $400 \mathrm{~nm}$ size were used for NSL. Ag, Au, and Al were used for NT formation. (b) A typical SEM image of the InSe sample after Ag NT deposition. Silicon with native oxide was used as substrate for SEM to avoid charging effects. (c), (d) optical images and (e), (f) AFM topographies of one typical InSe sample with different thicknesses before and after NSL with Ag, respectively. Inset of (e) displays the PL spectra of 7L InSe with a Voigt fitting. The red dashed lines in (f) marks the area of the thin flake as shown in the optical images. (g) Resonant and non-resonant Raman spectra acquired with $2.41 \mathrm{eV}$ and $1.94 \mathrm{eV}$, respectively, of $7 \mathrm{~L}$ InSe. Acquisition time for the Raman measurements were $10 \mathrm{~s} \times 2$ and $30 \mathrm{~s} \times 10$, respectively, and the laser power in both measurements were $\sim 100 \mu \mathrm{W}$. (h) Elongation patterns of the phonon vibrations visible in the Raman spectra. (i) Raman spectra of 7L InSe before and after NSL with Ag acquired with $1.58 \mathrm{eV}, 1.94 \mathrm{eV}$, and $2.33 \mathrm{eV}$ excitations. The broad spectral feature between 150 and $190 \mathrm{~cm}-1$ is due to the presence of E2g, and contribution of In203 (green dashed line and asterisk). The grey asterisk indicates a feature assigned to amorphous Se. Acquisition time for the measurements were $5 \mathrm{~s} \times 2$ and the laser power was $10 \mu \mathrm{W}$. 


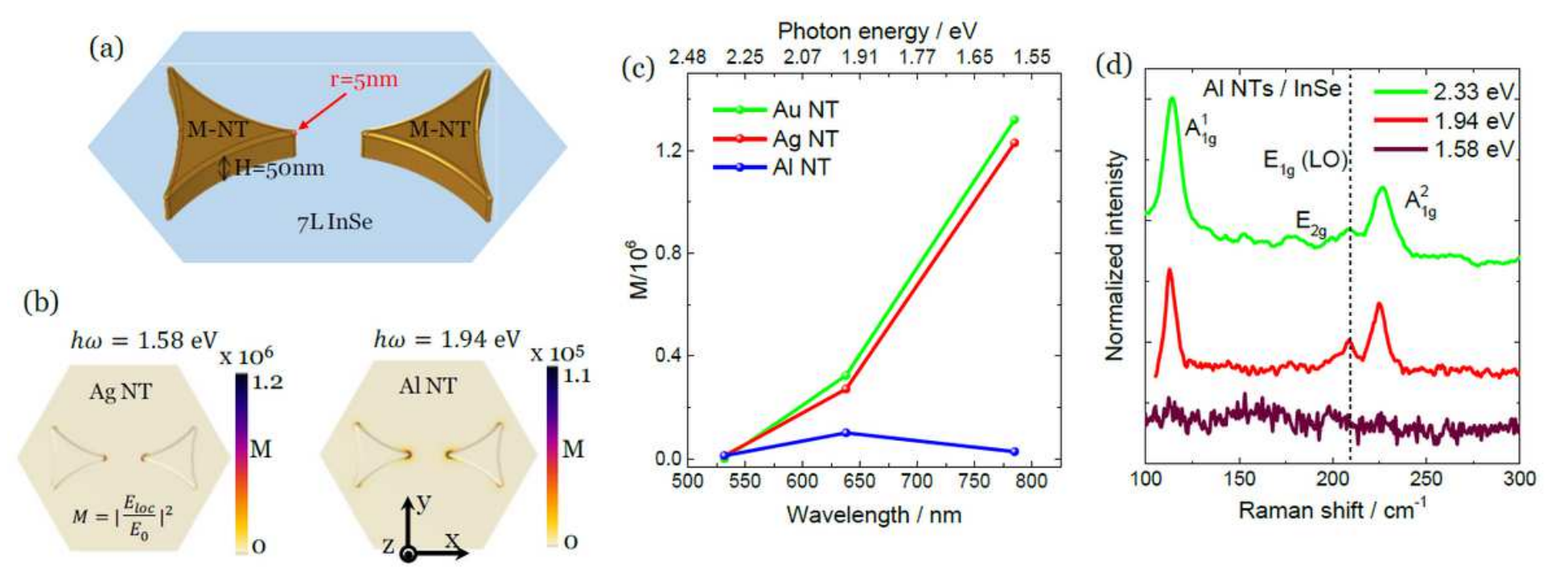

Figure 2

(a) Sketch of the model used in the FEM simulation of InSe/metal NTs. (b) Simulated electric field intensity M distribution at two different wavelengths for Ag and Al NTs. Simulated transmittance spectra of InSe/Ag, Au, and Al NTs systems for the spectral range from 380 to $1200 \mathrm{~nm}$ together with M distribution for Au NTs at $785 \mathrm{~nm}$ excitation are presented in SI3. (c) Absolute value of calculated maximum $\mathrm{M}$ at three different wavelengths. A similar plasmonic behaviour is expected for Ag and Au NTs on InSe for three selected wavelengths while InSe with Al NTs shows a maximum at $638 \mathrm{~nm}$ excitation. (d) Raman spectra of $7 \mathrm{~L}$ InSe with Al NTs acquired under three different wavelengths. As expected from simulations we can resolve the polar modes of InSe best at $638 \mathrm{~nm}(1.94 \mathrm{eV})$ excitation. Raman spectra of InSe with Au NTs are presented in SI3.
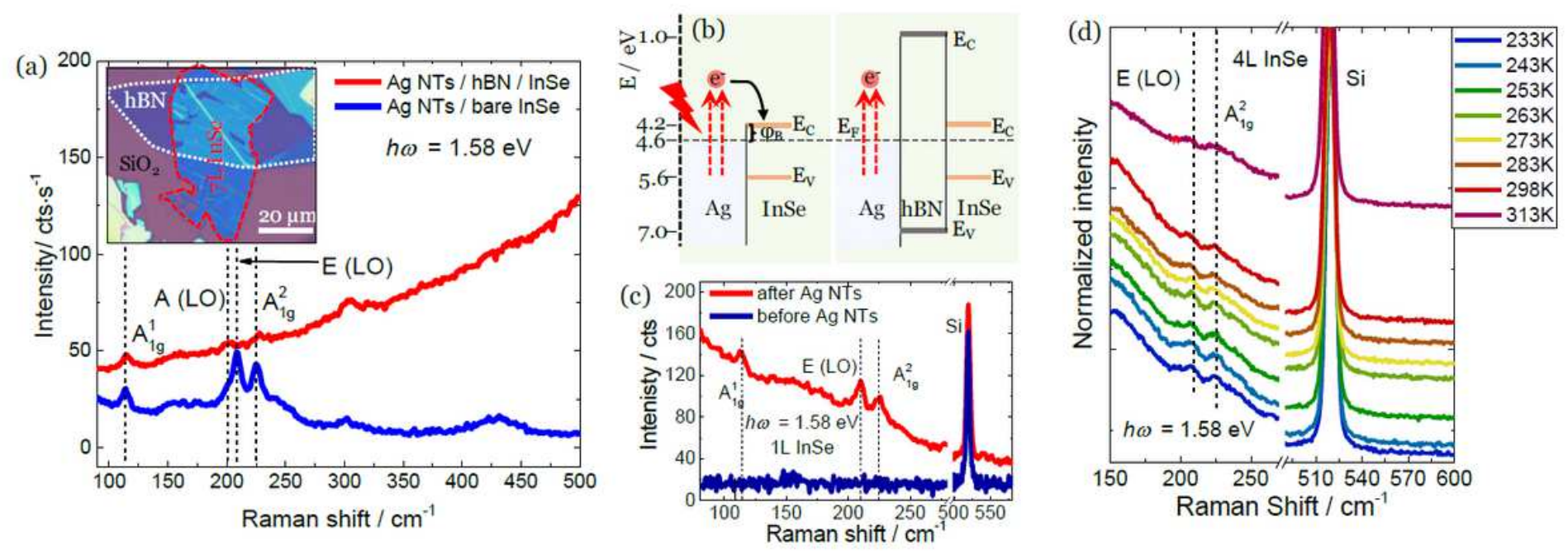

\section{Figure 3}

(a) Raman spectra taken with $1.58 \mathrm{eV}$ excitation of hBN encapsulated and bare InSe/Ag NTs demonstrating the influence of plasmonic hot electron doping. The inset is the optical image of the 
partially encapsulated sample used for the measurements. The areas marked by the white dotted and red dashed lines indicate hBN and InSe, respectively. (b) Simplified diagram of the band alignment illustrating the hot electron doping process. Left panel: InSe with Ag NTs, right panel: InSe/hBN/Ag NTs. The values are taken from the refs.13, 43, 44 (c) Raman spectra of 1L InSe before and after Ag NTs deposition. The bandgap of $1 \mathrm{~L}$ InSe is well above the excitation energy at $1.58 \mathrm{eV}$. (d) Temperature dependent Raman measurements of $4 \mathrm{~L}$ InSe/Ag NTs at $1.58 \mathrm{eV}$ excitation. The acquisition parameters of the Raman measurements are $30 \mathrm{~s} \times 2$ (7L) and $150 \sec \times 2(1 \mathrm{~L}), 10 \mu \mathrm{W}$ under 100x, 0.9 NA objective. The acquisition parameters for the temperature dependent Raman measurements were $150 \sec \times 2,10 \mu \mathrm{W}$ under 50x, 0.45 NA objective.

(a)

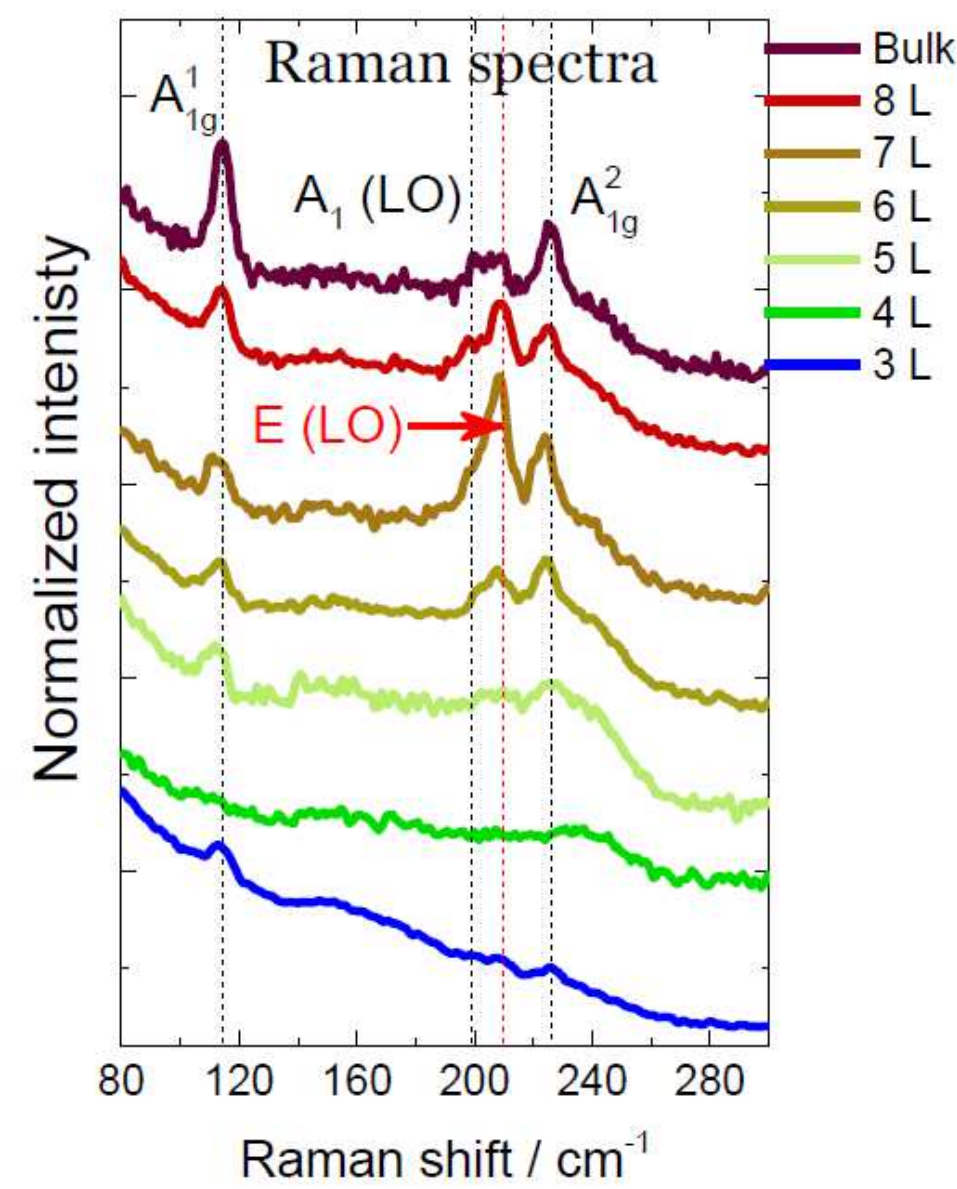

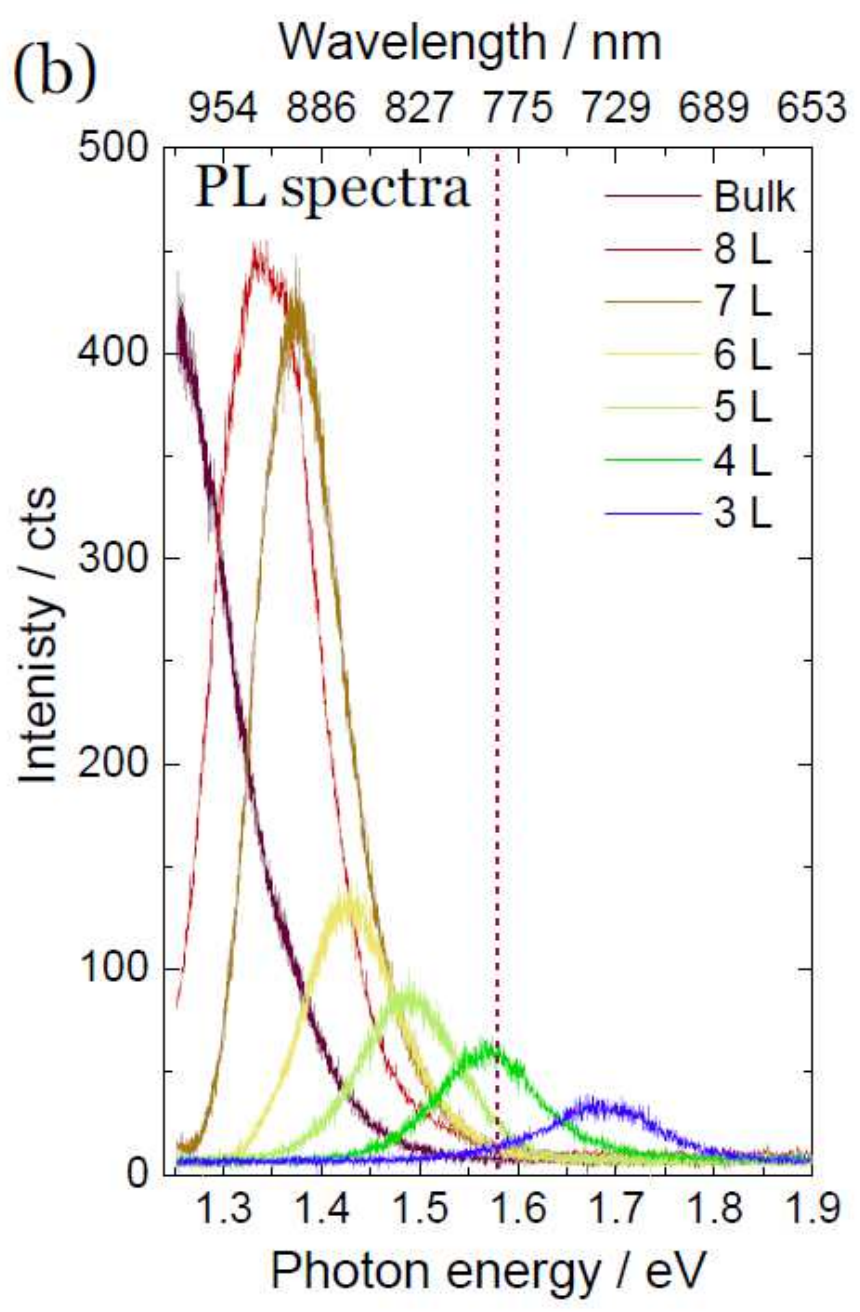

Figure 4

Layer dependent (a) Raman excited at $1.58 \mathrm{eV}$ and (b) corresponding PL spectra of InSe. There is a drastic drop of intensity of the polar $\mathrm{LO}$ modes from $7 \mathrm{~L}$ to $6 \mathrm{~L}$ corresponding to the thickness where the direct to indirect bandgap transition of InSe occurs.

\section{Supplementary Files}


This is a list of supplementary files associated with this preprint. Click to download.

- Supplementarylnformation.pdf 